Теорія Ймовір. та Матем. Статист. Вип. 69, 2003
Theor. Probability and Math. Statist.

No. 69, 2004, Pages 1-15

S 0094-9000(05)00609-5

Article electronically published on February 7, 2005

\title{
CATALYTIC BRANCHING RANDOM WALK AND QUEUEING SYSTEMS WITH RANDOM NUMBER OF INDEPENDENT SERVERS
} UDC 519.21

\author{
V. A. VATUTIN, V. A. TOPCHII , AND E. B. YAROVAYA
}

\begin{abstract}
A continuous time branching random walk on the lattice $\mathbf{Z}$ in which particles may produce children only at the origin is considered. Assuming that the underlying random walk is symmetric and the offspring reproduction law is critical, we find the asymptotic behavior of the survival probability of the process at time $t$ as $t \rightarrow \infty$ and the probability that the number of particles at the origin at time $t$ is positive. We also prove a Yaglom type conditional limit theorem for the total number of particles existing at time $t$. A relation between the model considered and a queueing system with a random number of independently operating servers is discussed.
\end{abstract}

\section{INTRODUCTION}

We consider the following modification of a standard branching random walk on $\mathbf{Z}$. Let a population of particles evolves as follows. The population is initiated at time $t=0$ by a single particle. Being outside of the origin the particle performs a continuous time random walk on $\mathbf{Z}$ with the infinitesimal transition matrix $A=|a(x, y)|_{x, y \in \mathbf{Z}}, a(0,0)<0$, until the moment when it hits the origin. At the origin it spends an exponentially distributed time with parameter 1 and then either jumps to a point $y \neq 0$ with probability $-(1-\alpha) a(0, y) a^{-1}(0,0) \stackrel{\text { def }}{=}(1-\alpha) \pi_{y}$, or dies with probability $\alpha$ producing just before its death a random number of children $\xi$ in accordance with the offspring generating function

$$
f(s) \stackrel{\text { def }}{=} \mathrm{E} s^{\xi}=\sum_{k=0}^{\infty} f_{k} s^{k} .
$$

The new particles behave independently and stochastically in the same way as the parent particle.

Now we describe our basic hypotheses on the characteristics of the process:

Hypothesis (I). The underlying random walk is symmetric, homogeneous, and irreducible on $\mathbf{Z}: a(x, y)=a(y, x), a(x, y)=a(0, y-x) \stackrel{\text { def }}{=} a(y-x)$ with $a(x) \geq 0, x \neq 0$, $a(0)<0$ and $\sum_{x \in \mathbf{Z}} a(x)=0$; in addition

$$
b^{2} \stackrel{\text { def }}{=} \sum_{x \in \mathbf{Z}} x^{2} a(x)<\infty .
$$

Hypothesis (II). The offspring generating function $f(s)$ is critical $\left(f^{\prime}(1)=1\right)$ and $\sigma^{2} \stackrel{\text { def }}{=} f^{\prime \prime}(1) \in(0, \infty)$.

2000 Mathematics Subject Classification. Primary 60J80, 60J15; Secondary 60K25, 60K05.

Supported by the RFBR grants 02-01-00266, 00-15-96136 and the INTAS grants 99-01317, 00-0265. 
Let $\zeta(t)$ denote the number of particles in the process at time $t$ at the origin, $\mu(t)$ the number of particles in the process at time $t$ outside the origin, and let

$$
\eta(t)=\zeta(t)+\mu(t)
$$

be the total number of particles in the process at time $t$. The goal of the present paper is to study the asymptotic behavior of the probabilities $Q(t)=\mathrm{P}(\mu(t)>0)$ and $q(t)=\mathrm{P}(\zeta(t)>0)$ and to establish a Yaglom type conditional limit theorem for $\eta(t)$.

Let

$$
K \stackrel{\text { def }}{=} \frac{2^{3 / 4}}{\sigma \pi^{1 / 4}} \sqrt{\frac{b(1-\alpha)}{\alpha}} .
$$

Our main results are contained in the following two theorems.

Theorem 1. Let Hypotheses (I) and (II) hold. Then

$$
Q(t)=\mathrm{P}(\mu(t)>0) \sim K t^{-1 / 4}, \quad t \rightarrow \infty,
$$

and for any $s \in[0,1]$,

$$
\lim _{t \rightarrow \infty} \mathrm{E}\left[s^{\mu(t)} \mid \eta(t)>0\right]=1-\sqrt{1-s} .
$$

Theorem 2. Under the conditions of Theorem 1,

$$
q(t)=\mathrm{P}(\zeta(t)>0) \sim \frac{K^{2} \pi}{\sqrt{t} \ln t}, \quad t \rightarrow \infty .
$$

The following assertion is an easy consequence of the previous theorems.

Corollary 3. Under the conditions of Theorem 1,

$$
\mathrm{P}(\eta(t)>0) \sim K t^{-1 / 4}, \quad t \rightarrow \infty,
$$

and for any $s \in[0,1]$

$$
\lim _{t \rightarrow \infty} \mathrm{E}\left[s^{\eta(t)} \mid \eta(t)>0\right]=1-\sqrt{1-s} .
$$

This model was first considered in [1, 2, 4, 5] in a more general setting, namely, for the case where particles walk on the $d$-dimensional lattice $\mathbf{Z}^{d}$. In these papers, the authors deduce basic equations for the probability generating functions of the random variables $\eta(x ; t)$, the total number of particles in the population at time $t$, and $\eta(x, y ; t)$, the number of particles at point $y \in \mathbf{Z}^{d}$ at time $t$, given that the population is initiated at time zero by a single particle located at point $x \in \mathbf{Z}^{d}$. Besides, those papers contain asymptotic representations for $\mathrm{E} \eta^{k}(x ; t)$ and $\mathrm{E} \eta^{k}(x, y ; t), k=1,2, \ldots$, in all dimensions. A superprocess version of this model, called the catalytic superprocess, is considered in 6. 8, 9].

These results can be applied in a natural way to the following queueing model.

Denote by $M(\lambda)$ a random variable having the exponential distribution with parameter $\lambda$.

Our model of the queueing system corresponds to a catalytic branching random walk on the lattice of integers with symmetric \pm 1 random walk (or on the half-line $y \in \mathbf{Z}^{+}$).

This system consists of a countable number of servers. Each server can be in one of the four states: busy, idle, broken or locked.

I. For each busy server:

1) the arrival process is Poisson with $\lambda=1 / 2$ and is independent of the arrival processes for the remaining servers (busy or idle);

2) service times are $M(1 / 2)$;

3) all customers arriving at a particular busy server stay in the queue to this server. 
II. Each idle server:

1 ) is waiting for the first customer for a time $\eta$, which is an $M(\alpha)$ random variable;

2) the arrival process of customers is Poisson with parameter $1-\alpha$;

3 ) if the arrival time $\tau_{1}$ of the first customer does not exceed $\eta$, the server starts to serve the customer with service time $M(1 / 2)$ and perform the service of this and subsequent customers according to the service discipline mentioned until it is idle again;

4) if $\tau_{1}>\eta$, then just after the deadline moment for its waiting time the server becomes broken and simultaneously $\xi$ new servers are unlocked, that is, they are in the operational state and idle from this moment on (note that $\xi=0$ with probability $f_{0}$ ).

III. At the initial moment $t=0$ we have one idle server and the remaining servers are locked.

It is not difficult to check that if, in terms of the catalytic branching random walk described above, the negative values on the lattice $\mathbf{Z}$ are identified with the positive ones and each particle on $\mathbf{Z}$ is associated with a server, then the total amount of particles is the number of servers in the system, while the coordinates of the particles on $\mathbf{Z}$ (or, to be more precise, on $\mathbf{Z}^{+}$) are the queue lengths at the corresponding servers.

Thus, Theorems 1 and 2 and Corollary 3 deal with the total number of busy servers and the total number of servers in the operational state at the moment $t$ and with probability that there are idle servers in the system at this moment.

\section{Auxiliary Results}

We temporarily forget that our random walk has a point of catalysis and consider an ordinary random walk on $\mathbf{Z}$ satisfying Hypothesis (I).

Let $p(t ; x, y)$ be the transition probability of the random walk, that is, the probability that starting at time $t=0$ at point $x$ the particle is located at point $y$ at time $t$. Under the conditions of Hypothesis (I) $p(t ; x, y)=p(t ; 0, y-x) \stackrel{\text { def }}{=} p(t ; y-x)$ and the backward Kolmogorov equations for $p(t ; x, y)$ are as follows:

$$
\frac{\partial p(t ; x)}{\partial t}=\sum_{y \in \mathbf{Z}} a(y) p(t ; y-x), \quad p(0 ; x)=\delta_{0}(x)
$$

where $\delta_{0}(x)=1$ if $x=0$, and $\delta_{0}(x)=0$ otherwise.

Lemma 4. Let Hypothesis (I) be valid. Then the function

$$
\phi(\theta) \stackrel{\text { def }}{=} \sum_{x \in \mathbf{Z}} a(x) e^{i \theta x}, \quad \theta \in[-\pi, \pi],
$$

is twice differentiable, real-valued, and nonpositive. It has a unique maximum on $[-\pi, \pi]$ at the point $\theta_{0}=0$, and there exists a constant $\gamma>0$ such that $\phi(\theta) \leq-\gamma \theta^{2}$ in $a$ neighborhood of zero.

Proof. Since $a(0)=-\sum_{x \neq 0} a(x)$ and the random walk is symmetric, we have

$$
\phi(\theta)=a(0)+\frac{1}{2} \sum_{x \neq 0} a(x)\left(e^{i \theta x}+e^{-i \theta x}\right)=\sum_{x \neq 0} a(x)(\cos \theta x-1) \leq 0 .
$$

The remaining part of the lemma follows from (2) and the irreducibility of the random walk.

For brevity we use the notation $p(t) \stackrel{\text { def }}{=} p(t ; 0,0)$. 
Lemma 5. Let Hypothesis (I) be valid. Then $p(t)$ and $-p^{\prime}(t)$ are monotone decreasing in $t$ and admit the representations

$$
\begin{aligned}
p(t) & \sim \gamma_{1} t^{-1 / 2}, \quad t \rightarrow \infty, \\
-p^{\prime}(t) & \sim \frac{\gamma_{1}}{2} t^{-3 / 2}, \quad t \rightarrow \infty,
\end{aligned}
$$

where

$$
\gamma_{1} \stackrel{\text { def }}{=} \frac{1}{b \sqrt{2 \pi}} .
$$

Proof. Let $\tilde{p}(t ; \theta) \stackrel{\text { def }}{=} \sum_{x \in \mathbf{Z}} p(t ; x) e^{i \theta x}$. Then (4) can be rewritten as follows:

$$
\frac{\partial \tilde{p}(t ; \theta)}{\partial t}=\phi(\theta) \tilde{p}(t ; \theta), \quad \tilde{p}(0 ; \theta)=1 .
$$

Hence $\tilde{p}(t ; \theta)=e^{t \phi(\theta)}$ and therefore

$$
p(t, x)=\frac{1}{2 \pi} \int_{-\pi}^{\pi} e^{t \phi(\theta)-i x \theta} d \theta .
$$

Thus

$$
\begin{gathered}
p(t)=\frac{1}{2 \pi} \int_{-\pi}^{\pi} e^{t \phi(\theta)} d \theta, \quad p^{\prime}(t)=\frac{1}{2 \pi} \int_{-\pi}^{\pi} \phi(\theta) e^{t \phi(\theta)} d \theta, \\
p^{\prime \prime}(t)=\frac{1}{2 \pi} \int_{-\pi}^{\pi} \phi^{2}(\theta) e^{t \phi(\theta)} d \theta .
\end{gathered}
$$

Since $\phi(\theta) \leq 0, \theta \in[-\pi, \pi]$, the desired monotonicity of $p(t)$ and $-p^{\prime}(t)$ follows. To complete the proof it remains to note that in view of Lemma 4 we can apply the Laplace method to the integral

$$
p(t)=\frac{1}{2 \pi} \int_{-\pi}^{\pi} e^{t \phi(\theta)} d \theta
$$

to get (5) and (6) (see, for instance, [10], Chapter 3, Section 7, Theorem 7.1).

Now we assume that the random walk starts at the origin at time $t=0$. Let $\tau_{1}$ be the time spent by the particle at the origin until it leaves the origin, and let $\tau_{2}$ be the time spent by this particle outside the origin until its first return to the origin. Set $G_{1}(t) \stackrel{\text { def }}{=} \mathrm{P}\left(\tau_{1} \leq t\right)=1-e^{-t}$ and $G_{2}(t) \stackrel{\text { def }}{=} \mathrm{P}\left(\tau_{2} \leq t\right)$.

In the sequel the following convention is used: for a function $g(t), t \geq 0$, we set

$$
\hat{g}(\lambda) \stackrel{\text { def }}{=} \int_{0}^{\infty} e^{-\lambda t} g(t) d t
$$

if, in addition, $g(t)$ is nonnegative and monotone increasing, then

$$
\check{g}(\lambda) \stackrel{\text { def }}{=} \int_{0}^{\infty} e^{-\lambda t} d g(t) .
$$

Lemma 6. Let Hypothesis (I) be valid. Then

$$
1-G_{2}(t) \sim \frac{b \sqrt{2}}{\sqrt{\pi t}}, \quad t \rightarrow \infty .
$$

Proof. It is not difficult to check that

$$
p(t)=1-G_{1}(t)+\int_{0}^{t} p(t-u) d\left(G_{1} * G_{2}(u)\right),
$$

where $*$ denotes the convolution. Using this relation we obtain

$$
\hat{p}(\lambda)=\widehat{1-G_{1}}(\lambda)+\hat{p}(\lambda) \check{G}_{1}(\lambda) \check{G}_{2}(\lambda)
$$


or

$$
\hat{p}(\lambda)\left(1-\check{G}_{1}(\lambda) \check{G}_{2}(\lambda)\right)=\widehat{1-G_{1}}(\lambda) .
$$

Recalling that $\check{G}_{1}(\lambda)=(1+\lambda)^{-1}$ we get

$$
\hat{p}(\lambda)\left(1-\frac{1}{1+\lambda} \check{G}_{2}(\lambda)\right)=\frac{1}{1+\lambda} \quad \text { and } \quad\left(\widehat{1-G_{2}}\right)(\lambda)=\frac{1-\lambda \hat{p}(\lambda)}{\lambda \hat{p}(\lambda)} .
$$

Since

$$
\hat{p}(\lambda) \sim \frac{\gamma_{1} \sqrt{\pi}}{\sqrt{\lambda}}, \quad \lambda \rightarrow+0,
$$

in view of Lemma 5 and a Tauberian theorem (Theorem 2 in [7], Chapter XIII, §5), we have

$$
\left(\widehat{1-G_{2}}\right)(\lambda) \sim \frac{1}{\lambda \hat{p}(\lambda)} \sim \frac{1}{\gamma_{1} \sqrt{\pi} \sqrt{\lambda}}, \quad \lambda \rightarrow+0
$$

Applying the Tauberian theorem once again we get

$$
1-G_{2}(t) \sim \frac{1}{\gamma_{1} \pi \sqrt{t}}=\frac{b \sqrt{2}}{\sqrt{\pi t}}, \quad t \rightarrow \infty
$$

(recall the definition of $\gamma_{1}$ in $(7)$ ).

Since $p(0)=1$, we can regard $G(t) \stackrel{\text { def }}{=} 1-p(t)$ as a distribution function. For $\alpha \in(0,1)$ let $U_{\alpha}(t)=\sum_{k=0}^{\infty} \alpha^{k} G^{* k}(t)$ and let $P(t)$ be the solution of the renewal equation

$$
P(t)=1-G_{1}(t)+\int_{0}^{t} P(t-u) d G_{3}(u)
$$

with

$$
G_{3}(t) \stackrel{\text { def }}{=} \alpha G_{1}(t)+(1-\alpha) G_{1} * G_{2}(t) .
$$

It is clear that

where

$$
P(t)=\left(1-G_{1}(\cdot)\right) * U(t)
$$

$$
U(t) \stackrel{\text { def }}{=} \sum_{k=0}^{\infty} G_{3}^{* k}(t)
$$

Lemma 7. We have

$$
P(t)=\frac{1}{\alpha}-\frac{1-\alpha}{\alpha} U_{\alpha}(t)
$$

Proof. Applying the Laplace transform to both sides of (10) we get

or

$$
\hat{P}(\lambda)=\frac{\left(\widehat{1-G_{1}}\right)(\lambda)}{\alpha\left(1-\check{G}_{1}(\lambda)\right)+(1-\alpha)\left(1-\check{G}_{1}(\lambda) \check{G}_{2}(\lambda)\right)}
$$

$$
\hat{P}(\lambda)=\frac{\hat{p}(\lambda)\left(\widehat{1-G_{1}}\right)(\lambda)}{\alpha\left(1-\check{G}_{1}(\lambda)\right) \hat{p}(\lambda)+(1-\alpha)\left(\widehat{1-G_{1}}\right)(\lambda)}
$$

in view of $(8)$. Hence, observing that $1-\check{G}_{1}(\lambda)=\lambda\left(\widehat{1-G_{1}}\right)(\lambda)$ we deduce

$$
\begin{aligned}
\hat{P}(\lambda) & =\frac{\hat{p}(\lambda)\left(\widehat{1-G_{1}}\right)(\lambda)}{\alpha \lambda\left(\widehat{1-G_{1}}\right)(\lambda) \hat{p}(\lambda)+(1-\alpha)\left(\widehat{1-G_{1}}\right)(\lambda)} \\
& =\frac{\hat{p}(\lambda)}{\alpha \lambda \hat{p}(\lambda)+(1-\alpha)}=\frac{\hat{p}(\lambda)}{1-\alpha(1-\lambda \hat{p}(\lambda))}=\frac{\hat{p}(\lambda)}{1-\alpha \check{G}(\lambda)} .
\end{aligned}
$$


On the other hand,

$$
\begin{aligned}
\int_{0}^{\infty} e^{-\lambda t}\left(\frac{1}{\alpha}-\frac{1-\alpha}{\alpha} U_{\alpha}(t)\right) d t & =\frac{1}{\lambda \alpha}-\frac{1-\alpha}{\alpha} \frac{1}{1-\alpha \check{G}(\lambda)} \\
& =\frac{1-\check{G}(\lambda)}{\lambda(1-\alpha \check{G}(\lambda))}=\frac{\hat{p}(\lambda)}{1-\alpha \check{G}(\lambda)} .
\end{aligned}
$$

Combining (14) and (15) we prove (13).

Lemma 8. $P(t)$ is a monotone decreasing function and

$$
P(t) \sim \frac{1}{1-\alpha} p(t) \sim \frac{\gamma_{1}}{1-\alpha} t^{-1 / 2}, \quad t \rightarrow \infty .
$$

In addition, there exists a constant $C>0$ such that

$$
\left|P^{\prime}(t)\right| \leq C(t+1)^{-3 / 2}
$$

for all $t \geq 0$.

Proof. The monotonicity of $P(t)$ is a trivial consequence of (13). To establish the remaining statements of the lemma observe that $1-G(t)=p(t) \sim \gamma_{1} t^{-1 / 2}$ as $t \rightarrow \infty$. It is shown in (3], Chapter IV, Section 4) that under this condition

$$
U_{\alpha}(t)=\frac{1}{1-\alpha}-\frac{\alpha}{(1-\alpha)^{2}} p(t)+o(p(t)), \quad t \rightarrow \infty .
$$

This equality and the previous lemma complete the proof of (16). To prove inequality (17) we need more delicate arguments. First observe that

$$
P^{\prime}(t)=-\frac{1-\alpha}{\alpha} U_{\alpha}^{\prime}(t)=-\frac{1-\alpha}{\alpha} \sum_{k=1}^{\infty} \alpha^{k} g^{* k}(t), \quad t>0
$$

where

$$
g^{* k}(t) \stackrel{\text { def }}{=}\left(G^{* k}(t)\right)^{\prime}=\int_{0}^{t} g^{*(k-1)}(t-u) g(u) d u
$$

is the $k$ th convolution of the density $g(t)$ of $G(t)$. Note that $g(t)=-p^{\prime}(t)$ and therefore $g(t)$ is monotone decreasing by Lemma 5 . Consequently, for any $k>1$

$$
\begin{aligned}
g^{* k}(t) & \leq g\left(\frac{t}{k}\right) \int_{t k^{-1}}^{t} g^{*(k-1)}(t-u) d u+g^{*(k-1)}\left(t \frac{k-1}{k}\right) \int_{0}^{t k^{-1}} g(u) d u \\
& \leq g\left(\frac{t}{k}\right)+g^{*(k-1)}\left(t \frac{k-1}{k}\right) \leq 2 g\left(\frac{t}{k}\right)+g^{*(k-2)}\left(t \frac{k-2}{k}\right) \leq k g\left(\frac{t}{k}\right) .
\end{aligned}
$$

Using Lemma 5 once more we obtain that

$$
g(t)=-p^{\prime}(t) \sim \frac{\gamma_{1}}{2} t^{-3 / 2}, \quad t \rightarrow \infty,
$$

whence $g(t)$ is bounded by (4) with $x=0$. As a result we get that there exists a constant $c>0$ such that

for all $t \geq 0$. Hence it follows that

$$
g(t) \leq \frac{c}{(t+1)^{3 / 2}}
$$

$$
g^{* k}(t) \leq k g\left(\frac{t}{k}\right) \leq \frac{c k^{5 / 2}}{(t+k)^{3 / 2}} \leq c k^{5 / 2}(t+1)^{-3 / 2}, \quad t \geq 0 .
$$

Substituting this estimate into (18) we get

$$
\left|P^{\prime}(t)\right| \leq c(t+1)^{-3 / 2} \sum_{k=1}^{\infty} k^{5 / 2} \alpha^{k} \stackrel{\text { def }}{=} C(t+1)^{-3 / 2},
$$


since $\sum_{k=1}^{\infty} k^{5 / 2} \alpha^{k}<\infty$ in view of $\alpha<1$. This completes the proof of (17).

\section{BRANChing RANDOM WALK AND Bellman-HARRIS PROCESSES}

In this section we prove Theorem 1 by introducing an auxiliary Bellman-Harris branching process with two types of particles.

A Bellman-Harris branching process with two types of particles can be described as follows. It is initiated by a single particle of type $i, i=1,2$. This particle has a random life time with a distribution function $G_{i}(t)$. When dying this particle produces children according to an offspring generating function $f_{i}\left(s_{1}, s_{2}\right)$. The new particles of type $j=1,2$ evolve independently with the life time distribution $G_{j}(t)$ and offspring generating function $f_{j}\left(s_{1}, s_{2}\right)$. Let $M=\left\|\frac{\partial f_{i}}{\partial s_{j}}(1,1)\right\|_{i, j=1,2}$ be the mean matrix of the process. The process is called critical indecomposable if the maximum (in the absolute value) eigenvalue of $M$ (the Perron root of $M$ ) equals 1 and there is an integer $n$ such that all entries of $M^{n}$ are positive.

Let $v=\left(v_{1}, v_{2}\right)$ and $u=\left(u_{1}, u_{2}\right)$ be the left and right positive eigenvectors corresponding to the Perron root of $M$ and such that $u_{1}+u_{2}=1$ and $v_{1} u_{1}+v_{2} u_{2}=1$. Denote by $Z_{i}(t), i=1,2$, the number of particles of type $i$ in this process at time $t$. Set

$$
F_{i}\left(t ; s_{1}, s_{2}\right)=\mathrm{E} s_{1}^{Z_{1}(t)} s_{2}^{Z_{2}(t)}, \quad i=1,2,
$$

for the probability generating functions of the number of particles of both types given that the process is initiated at time zero by a single particle of type $i$.

Now we are ready to recall a result from [12] concerning the critical Bellman-Harris branching processes adapted to the case of two types of particles.

Theorem 9 (see [12]). Let

$$
x-v_{1}\left(1-f_{1}\left(1-u_{1} x, 1-u_{2} x\right)\right)-v_{2}\left(1-f_{2}\left(1-u_{1} x, 1-u_{2} x\right)\right) \sim B^{2} x^{2}
$$

as $x \rightarrow+0$ with $B>0$. If there exist $\beta \in(0,1)$ and nonnegative constants $c_{1}$ and $c_{2}$ with $c_{1}+c_{2}>0$ such that $1-G_{i}(t)=c_{i} t^{-\beta}+o\left(t^{-\beta}\right)$ as $t \rightarrow \infty$, then

$$
1-F_{i}\left(t ; s_{1}, s_{2}\right) \sim u_{i} B^{-1} \sqrt{v_{1} c_{1}\left(1-s_{1}\right)+v_{2} c_{2}\left(1-s_{2}\right)} t^{-\beta / 2}, \quad t \rightarrow \infty
$$

and

$$
\begin{aligned}
\lim _{t \rightarrow \infty} \mathrm{E} & {\left[s_{1}^{Z_{1}(t)} s_{2}^{Z_{2}(t)} \mid Z_{1}(t)+Z_{2}(t)>0 ; Z_{1}(0)=1, Z_{2}(0)=0\right] } \\
& =1-\sqrt{\frac{v_{1} c_{1}\left(1-s_{1}\right)+v_{2} c_{2}\left(1-s_{2}\right)}{v_{1} c_{1}+v_{2} c_{2}}}
\end{aligned}
$$

for all fixed $s_{1}, s_{2} \in[0,1)$.

We apply this theorem to the following critical Bellman-Harris process with two types of particles. A particle of the first type has the life time distribution

$$
G_{1}(t)=\mathrm{P}\left(\tau_{1} \leq t\right)=1-e^{-t}, \quad t \geq 0 ;
$$

when dying the particle produces the offspring of two types in accordance with the probability generating function $f_{1}\left(s_{1}, s_{2}\right)=\alpha f\left(s_{1}\right)+(1-\alpha) s_{2}$, that is, it produces with probability $\alpha f_{k}$ exactly $k$ particles of the first type and with probability $1-\alpha$ exactly one particle of the second type (recall the definition of $f(s)$ in $(1)$ ). The life time distribution of a particle of the second type is $G_{2}(t)=\mathrm{P}\left(\tau_{2} \leq t\right)$ (that is, it coincides in distribution with the time spent outside the origin by the parent particle of the catalytic branching random walk under investigation until the first return to the origin, provided that the initial particle is located at point 0 at time $t=0$ and it does not produce children during its first stay at 0 ). When dying a particle of the second type produces the offspring in accordance with the probability generating function $f_{2}\left(s_{1}, s_{2}\right)=s_{1}$, that is, it produces 
exactly one particle of the first type and nothing else. It is not difficult to understand that we have $\left(Z_{1}(t), Z_{2}(t)\right) \stackrel{\text { distr }}{=}(\zeta(t), \mu(t))$ for the process constructed in this way.

Now to prove Theorem 1 it suffices to apply Theorem 9 to $\left(Z_{1}(t), Z_{2}(t)\right)$. It follows from the description above that in this case

$$
M=\left(\begin{array}{cc}
\alpha & 1-\alpha \\
1 & 0
\end{array}\right), \quad v_{1}=\frac{2}{2-\alpha}, \quad v_{2}=\frac{2(1-\alpha)}{2-\alpha}, \quad u_{1}=u_{2}=\frac{1}{2},
$$

and therefore

$$
\begin{aligned}
x-v_{1} & \left(1-f_{1}\left(1-u_{1} x, 1-u_{2} x\right)\right)-v_{2}\left(1-f_{2}\left(1-u_{1} x, 1-u_{2} x\right)\right) \\
& =x-\frac{2}{2-\alpha}\left(1-f_{1}\left(1-\frac{x}{2}, 1-\frac{x}{2}\right)\right)-\frac{2(1-\alpha)}{2-\alpha}\left(1-f_{2}\left(1-\frac{x}{2}, 1-\frac{x}{2}\right)\right) \\
& =x-\frac{2}{2-\alpha}\left(\alpha\left(1-f\left(1-\frac{x}{2}\right)\right)+(1-\alpha) \frac{x}{2}\right)-\frac{2(1-\alpha)}{2-\alpha} \frac{x}{2} \\
& =\frac{2 \alpha}{2-\alpha}\left(\frac{x}{2}-\left(1-f\left(1-\frac{x}{2}\right)\right)\right) \\
& \sim \frac{2 \alpha}{2-\alpha} \frac{f^{\prime \prime}(1)}{2}\left(\frac{x}{2}\right)^{2}=\frac{\alpha}{2-\alpha} \frac{f^{\prime \prime}(1)}{4} x^{2}=\frac{\alpha}{2-\alpha} \frac{\sigma^{2}}{4} x^{2}=B^{2} x^{2}
\end{aligned}
$$

as $x \rightarrow+0$. Moreover $\beta=1 / 2$ and $c_{2}=b \sqrt{2 / \pi}$ by Lemma 6 , while $1-G_{1}(t)=o\left(t^{-1 / 2}\right)$ as $t \rightarrow \infty$ implies that $c_{1}=0$.

Using these relations in (19) with $s_{1}=s_{2}=0$ we get

$$
\begin{aligned}
\mathrm{P}(\eta(t)>0)= & \mathrm{P}(\zeta(t)+\mu(t)>0)=\mathrm{P}\left(Z_{1}(t)+Z_{2}(t)>0 ; Z_{1}(0)=1, Z_{2}(0)=0\right) \\
= & 1-F_{1}(t ; 0,0) \\
\sim & \frac{1}{2} \sqrt{\frac{2-\alpha}{\alpha}} \frac{2}{\sigma} \sqrt{\frac{2(1-\alpha)}{2-\alpha} b \sqrt{\frac{2}{\pi}}} t^{-1 / 4}=\frac{2^{3 / 4}}{\sigma \sqrt[4]{\pi}} \sqrt{\frac{b(1-\alpha)}{\alpha}} t^{-1 / 4}=K t^{-1 / 4}, \\
t \rightarrow \infty &
\end{aligned}
$$

and

$$
\lim _{t \rightarrow \infty} \mathrm{E}\left[s_{1}^{\zeta(t)} s_{2}^{\mu(t)} \mid \zeta(t)+\mu(t)>0\right]=1-\sqrt{\frac{v_{1} c_{1}\left(1-s_{1}\right)+v_{2} c_{2}\left(1-s_{2}\right)}{v_{1} c_{1}+v_{2} c_{2}}}=1-\sqrt{1-s_{2}} .
$$

This shows, in particular, that $\mathrm{P}(\eta(t)>0) \sim \mathrm{P}(\zeta(t)>0)$ as $t \rightarrow \infty$ and establishes (3), completing the proof of Theorem 1. Thus if the process with a catalyst at the origin survives, then there are "practically" no particles at the origin and, besides, conditioned on the survival up to time $t$, the limiting distribution of the particles located outside the origin is discrete.

\section{Particles at the origin}

The main goal of this section is to investigate the asymptotic behavior of the probability that there are particles at the origin at time $t$ as $t \rightarrow \infty$. Recall that under our assumption on $f_{i}\left(s_{1}, s_{2}\right), i=1,2$,

$F_{1}\left(t ; s_{1}, s_{2}\right)=s_{1}\left(1-G_{1}(t)\right)+\int_{0}^{t}\left(\alpha f\left(F_{1}\left(t-u ; s_{1}, s_{2}\right)\right)+(1-\alpha) F_{2}\left(t-u ; s_{1}, s_{2}\right)\right) d G_{1}(u)$

and

$$
F_{2}\left(t ; s_{1}, s_{2}\right)=s_{2}\left(1-G_{2}(t)\right)+\int_{0}^{t} F_{1}\left(t-u ; s_{1}, s_{2}\right) d G_{2}(u)
$$


(see [11, Chapter VIII, §1). Substituting the second of these equalities into the first one we get

$$
\begin{aligned}
F_{1}\left(t ; s_{1}, s_{2}\right)= & s_{1}\left(1-G_{1}(t)\right)+s_{2}(1-\alpha)\left(1-G_{2}(\cdot)\right) * G_{1}(t) \\
& +\int_{0}^{t} \alpha f\left(F_{1}\left(t-u ; s_{1}, s_{2}\right)\right) d G_{1}(u) \\
& +\int_{0}^{t}(1-\alpha) F_{1}\left(t-u ; s_{1}, s_{2}\right) d\left(G_{1} * G_{2}(u)\right) .
\end{aligned}
$$

In particular, letting $F(t ; s) \stackrel{\text { def }}{=} \mathrm{E} s^{\mu(t)}=F_{1}(t ; s, 1)$ we obtain

$$
\begin{aligned}
F(t ; s)= & s\left(1-G_{1}(t)\right)+(1-\alpha)\left(1-G_{2}(\cdot)\right) * G_{1}(t) \\
& +\int_{0}^{t} \alpha f(F(t-u ; s)) d G_{1}(u) \\
& +\int_{0}^{t}(1-\alpha) F(t-u ; s) d\left(G_{1} * G_{2}(u)\right) .
\end{aligned}
$$

Hence

$$
q(t ; s)=(1-s)\left(1-G_{1}(t)\right)+q(\cdot ; s) *\left(\alpha G_{1}+(1-\alpha) G_{1} * G_{2}\right)(t)-\alpha h(q(\cdot ; s)) * G_{1}(t),
$$

where $q(t ; s) \stackrel{\text { def }}{=} 1-F(t ; s)$ and $h(x) \stackrel{\text { def }}{=} 1-x-f(1-x)$. Let $q(t) \stackrel{\text { def }}{=} q(t ; 0)$. Then

$$
q(t)=1-G_{1}(t)+q(\cdot) * G_{3}(t)-\alpha h(q(\cdot)) * G_{1}(t)
$$

in view of (11). Solving this renewal equation with respect to $q(t)$ we see that

$$
q(t)=\left(1-G_{1}(\cdot)\right) * U(t)-\alpha h(q(\cdot)) * G_{1} * U(t),
$$

where $U(t)$ is the same as in (12). Since $G_{1}(t)=1-e^{-t}$, it follows that

$$
\left(G_{1} * U(t)\right)^{\prime}=\left(1-G_{1}(\cdot)\right) * U(t)=P(t) .
$$

This allows us to rewrite (21) as follows:

$$
q(t)=P(t)-\alpha \int_{0}^{t} h(q(t-u)) P(u) d u
$$

Now we are ready to start the proof of Theorem 2. We divide the proof into several steps. The first lemma gives an estimate from below for $q(t)=\mathrm{P}(\zeta(t)>0)$.

Lemma 10. Under the condition of Theorem 2 there exists a constant $C_{1}>0$ such that

$$
q(t)>\frac{C_{1}}{\sqrt{t} \ln t}
$$

for all sufficiently large $t$.

Proof. Set $A(t)=\mathrm{E} \zeta(t)$ and $B(t)=\mathrm{E} \zeta(t)(\zeta(t)-1)$. Differentiating (20) with respect to $s$ at the point $s=1$ and using $f^{\prime}(1)=1$ we get

$$
A(t)=1-G_{1}(t)+A * G_{3}(t)=\left(1-G_{1}(\cdot)\right) * U(t)=P(t)
$$

and

$$
\begin{aligned}
B(t) & =\alpha A^{2} * G_{1}(t)+B * G_{3}(t)=\alpha A^{2} * G_{1} * U(t) \\
& =\alpha \int_{0}^{t} P^{2}(t-u) d\left(G_{1} * U\right)(u)=\alpha \int_{0}^{t} P^{2}(t-u) P(u) d u .
\end{aligned}
$$

Using (16) we deduce

$$
B(t) \sim \frac{\alpha \gamma_{1}^{3}}{(1-\alpha)^{3}} \frac{\ln t}{\sqrt{t}}
$$


whence

$$
q(t)=\mathrm{P}(\zeta(t)>0) \geq \frac{\mathrm{E}^{2} \zeta(t)}{\mathrm{E} \zeta^{2}(t)} \sim \frac{1-\alpha}{\alpha \gamma_{1}} \frac{1}{\sqrt{t} \ln t}, \quad t \rightarrow \infty,
$$

which proves the lemma.

\section{Lemma 11.}

$$
\alpha \int_{0}^{\infty} h(q(u)) d u=1
$$

Proof. Applying the Laplace transform to both sides of (22) we get

$$
\hat{q}(\lambda)=\hat{P}(\lambda)(1-\alpha \hat{h}(\lambda))
$$

where we set

$$
\hat{h}(\lambda)=\int_{0}^{\infty} e^{-\lambda t} h(q(t)) d t .
$$

Since $\hat{h}(\lambda)$ is monotone increasing in $\lambda$ as $\lambda \rightarrow+0$, the limit $\lim _{\lambda \rightarrow+0} \alpha \hat{h}(\lambda)=c_{0} \leq 1$ exists and, in particular, $\int_{0}^{\infty} h(q(t)) d t<\infty$. Now we show that $c_{0}=1$. Indeed, assuming the contrary we obtain from (9) and (14) that

$$
\hat{q}(\lambda) \sim\left(1-c_{0}\right) \hat{P}(\lambda) \sim \frac{\left(1-c_{0}\right)}{1-\alpha} \frac{\gamma_{1} \sqrt{\pi}}{\sqrt{\lambda}}, \quad \lambda \rightarrow+0,
$$

whence

$$
\int_{0}^{t} q(u) d u \sim \frac{2\left(1-c_{0}\right)}{1-\alpha} \gamma_{1} \sqrt{t}, \quad t \rightarrow \infty
$$

Thus

$$
\int_{t}^{2 t} q(u) d u \sim(\sqrt{2}-1) \frac{2\left(1-c_{0}\right)}{1-\alpha} \gamma_{1} \sqrt{t}, \quad t \rightarrow \infty .
$$

On the other hand, by Hölder's inequality,

$$
\frac{1}{t}\left(\int_{t}^{2 t} q(u) d u\right)^{2} \leq \int_{t}^{2 t} q^{2}(u) d u \leq \frac{4}{f^{\prime \prime}(1)} \int_{t}^{2 t} h(q(u)) d u
$$

for large $t$, since $h(x)=1-x-f(1-x) \sim f^{\prime \prime}(1) x^{2} / 2$ as $x \rightarrow+0$.

Thus if $c_{0}<1$, then the left-hand side of the preceding relation tends to a positive constant as $t \rightarrow \infty$, while the right-hand side tends to zero. This contradiction shows that $c_{0}=1$ as desired.

Corollary 12. Under the conditions of Theorem 2

$$
q(t)=o(P(t)), \quad t \rightarrow \infty .
$$

Proof. Using (22) and Lemma 11 we have

$$
q(t) \leq P(t)\left(1-\alpha \int_{0}^{t} h(q(t-u)) d u\right)=\alpha P(t) \int_{t}^{\infty} h(q(u)) d u=o(P(t)),
$$

The next lemma is one of the crucial steps in the proof of Theorem 2.

Lemma 13. Under the conditions of Theorem 2 the function $L(\lambda) \stackrel{\text { def }}{=} \hat{q}(\lambda) / \hat{P}(\lambda)$ is slowly varying as $\lambda \rightarrow+0$. 
Proof. It follows from (23) that for any $c>0$ there exists a constant $C_{1}>0$ such that

$$
\begin{aligned}
L(\lambda)-L(c \lambda) & =\alpha(\hat{h}(\lambda)-\hat{h}(c \lambda))=\alpha \int_{0}^{\infty} e^{-\lambda t}\left(1-e^{-\lambda(c-1) t}\right) h(q(t)) d t \\
& \leq C_{1} \alpha \int_{0}^{\infty} e^{-\lambda t}\left(1-e^{-\lambda(c-1) t}\right) q^{2}(t) d t
\end{aligned}
$$

Our goal is to show that the right-hand side of $(24)$ is $o(L(\lambda))$ as $\lambda \rightarrow+0$. Put

$$
\begin{gathered}
I_{1}(\lambda) \stackrel{\text { def }}{=} \int_{0}^{1 / \lambda} e^{-\lambda t}\left(1-e^{-\lambda(c-1) t}\right) q^{2}(t) d t, \\
I_{2}(\lambda) \stackrel{\text { def }}{=} \int_{1 / \lambda}^{\infty} e^{-\lambda t}\left(1-e^{-\lambda(c-1) t}\right) q^{2}(t) d t .
\end{gathered}
$$

In view of the inequality $1-e^{-x} \leq x, x \geq 0$, we have

$$
\begin{aligned}
I_{1}(\lambda) & \leq \lambda(c-1) \int_{0}^{1 / \lambda} e^{-\lambda t} t q^{2}(t) d t \leq c \sqrt{\lambda} \int_{0}^{1 / \lambda} e^{-\lambda t} \sqrt{t} q^{2}(t) d t \\
& \leq c \sqrt{\lambda} \int_{0}^{\infty} e^{-\lambda t}(\sqrt{t} q(t)) q(t) d t .
\end{aligned}
$$

Note that

$$
\int_{0}^{\infty} e^{-\lambda t}(\sqrt{t} q(t)) q(t) d t=o\left(\int_{0}^{\infty} e^{-\lambda t} q(t) d t\right), \quad \lambda \rightarrow+0 .
$$

Indeed, by Lemma 10

$$
\int_{0}^{\infty} q(t) d t=\infty
$$

and by Corollary 12

$$
\sqrt{t} q(t)=o(\sqrt{t} P(t))=o(1), \quad t \rightarrow \infty .
$$

Hence the desired relation follows. Using the equivalence

$$
\hat{P}(\lambda) \sim \frac{1}{1-\alpha} \frac{\gamma_{1} \sqrt{\pi}}{\sqrt{\lambda}}, \quad \lambda \rightarrow+0,
$$

we get

$$
I_{1}(\lambda)=o\left(\sqrt{\lambda} \int_{0}^{\infty} e^{-\lambda t} q(t) d t\right)=o\left(\frac{\hat{q}(\lambda)}{\hat{P}(\lambda)}\right)=o(L(\lambda)), \quad \lambda \rightarrow+0 .
$$

On the other hand,

$$
\begin{aligned}
I_{2}(\lambda) & \leq \int_{1 / \lambda}^{\infty} e^{-\lambda t} q^{2}(t) d t \leq \sup _{u \geq \lambda^{-1}} q(u) \int_{1 / \lambda}^{\infty} e^{-\lambda t} q(t) d t \\
& =o\left(P(1 / \lambda) \int_{0}^{\infty} e^{-\lambda t} q(t) d t\right)=o(\sqrt{\lambda} \hat{q}(\lambda))=o(L(\lambda)), \quad \lambda \rightarrow+0 .
\end{aligned}
$$

Therefore

$$
\frac{L(\lambda c)}{L(\lambda)}=1+\frac{L(\lambda c)-L(\lambda)}{L(\lambda)} \rightarrow 1, \quad \lambda \rightarrow+0
$$

for all $c>1$ and this is just the definition of a slowly varying function (see [7, Chapter VIII, §8). 
Corollary 14. The integral

$$
\int_{t}^{\infty} h(q(u)) d u
$$

is a slowly varying function as $t \rightarrow \infty$.

Proof. Evidently,

$$
L(\lambda)=1-\alpha \int_{0}^{\infty} e^{-\lambda t} h(q(t)) d t=\lambda \alpha \int_{0}^{\infty} e^{-\lambda t}\left(\int_{t}^{\infty} h(q(u)) d u\right) d t,
$$

whence

$$
\alpha \int_{0}^{\infty} e^{-\lambda t}\left(\int_{t}^{\infty} h(q(u)) d u\right) d t=\frac{L(\lambda)}{\lambda}
$$

is a regularly varying function with parameter 1 as $\lambda \rightarrow+0$. Since

$$
\int_{t}^{\infty} h(q(u)) d u
$$

is monotone decreasing in $t$, Theorem 4 in [7], Chapter XIII, $\S 5$ yields

$$
\alpha \int_{t}^{\infty} h(q(u)) d u \sim L\left(t^{-1}\right), \quad t \rightarrow \infty,
$$

as required.

Corollary 15. We have

$$
\int_{0}^{t} q(u) d u \sim \frac{2 \gamma_{1}}{1-\alpha} \sqrt{t} L\left(t^{-1}\right)
$$

as $t \rightarrow \infty$.

Proof. By (23) and (25),

$$
\hat{q}(\lambda)=\hat{P}(\lambda)(1-\alpha \hat{h}(\lambda))=\hat{P}(\lambda) L(\lambda) \sim \frac{1}{1-\alpha} \frac{\gamma_{1} \sqrt{\pi}}{\sqrt{\lambda}} L(\lambda), \quad \lambda \rightarrow+0 .
$$

Hence the corollary follows by applying a Tauberian theorem ([7], Chapter XIII, §5).

Lemma 16. As $t \rightarrow \infty$,

$$
J(t) \stackrel{\text { def }}{=} \int_{0}^{t} h(q(u))(P(t-u)-P(t)) d u=o\left(P(t) L\left(t^{-1}\right)\right) .
$$

Proof. Fix a number $\varepsilon \in(0,1 / 2)$ and split $J(t)$ into three integrals:

$$
J(t)=J_{1}(t)+J_{2}(t)+J_{3}(t)
$$

where

$$
\begin{aligned}
& 0 \leq J_{1}(t) \stackrel{\text { def }}{=} \int_{t(1-\varepsilon)}^{t} h(q(u))(P(t-u)-P(t)) d u, \\
& 0 \leq J_{2}(t) \stackrel{\text { def }}{=} \int_{t \varepsilon}^{t(1-\varepsilon)} h(q(u))(P(t-u)-P(t)) d u, \\
& 0 \leq J_{3}(t) \stackrel{\text { def }}{=} \int_{0}^{t \varepsilon} h(q(u))(P(t-u)-P(t)) d u .
\end{aligned}
$$

Since $P(t)$ is monotone,

$$
J_{1}(t) \leq \int_{t(1-\varepsilon)}^{t} h(q(u)) P(t-u) d u=\int_{0}^{t \varepsilon} h(q(t-u)) P(u) d u .
$$


Recall that

$$
q(t) \leq P(t) \int_{t}^{\infty} h(q(u)) d u \leq c P(t) L\left(t^{-1}\right), \quad c>0 .
$$

Properties of slowly varying functions and the inequality $h(x) \leq \sigma^{2} x^{2}$ for sufficiently small $x>0$ imply by Lemma 8 that

$$
\begin{aligned}
J_{1}(t) & \leq \sup _{v \in[t(1-\varepsilon), t]} h(q(v)) \int_{0}^{t \varepsilon} P(u) d u \\
& \leq c_{1} P^{2}(t) L^{2}\left(t^{-1}\right) \int_{0}^{t \varepsilon} P(u) d u \leq c_{2} P^{2}(t) L^{2}\left(t^{-1}\right) \sqrt{\varepsilon t} \\
& \leq c_{3} P(t) L^{2}\left(t^{-1}\right) \sqrt{\varepsilon}=o\left(P(t) L\left(t^{-1}\right)\right), \quad t \rightarrow \infty,
\end{aligned}
$$

since $L\left(t^{-1}\right) \rightarrow 0$ as $t \rightarrow \infty$. Using Corollary 14 and properties of slowly varying functions we see that

$$
\begin{aligned}
J_{2}(t) & \leq P(t \varepsilon) \int_{t \varepsilon}^{t(1-\varepsilon)} h(q(u)) d u \\
& =O\left(P(t \varepsilon)\left(L\left((\varepsilon t)^{-1}\right)-L\left((1-\varepsilon) t^{-1}\right)\right)\right) \\
& =o\left(P(t) L\left(t^{-1}\right)\right), \quad t \rightarrow \infty .
\end{aligned}
$$

By the mean value theorem, Lemma 8 , and the Tauberian theorems,

$$
\begin{aligned}
J_{3}(t) & \leq \sup _{v \in[t(1-\varepsilon), t]}\left|P^{\prime}(v)\right| \int_{0}^{t \varepsilon} u h(q(u)) d u \leq c t^{-3 / 2} \int_{0}^{t \varepsilon} u q^{2}(u) d u \\
& \leq c_{1} t^{-3 / 2} \int_{0}^{t \varepsilon} u P^{2}(u) L^{2}\left(u^{-1}\right) d u \leq c_{2} t^{-3 / 2} \int_{0}^{t \varepsilon} L^{2}\left(u^{-1}\right) d u \\
& \leq c_{3} t^{-3 / 2} t \varepsilon L^{2}\left((\varepsilon t)^{-1}\right)=o\left(P(t) L\left(t^{-1}\right)\right), \quad t \rightarrow \infty
\end{aligned}
$$

Combining (26)-(28) we complete the proof of Lemma 16.

Corollary 17. Under the conditions of Theorem 2

$$
q(t)=P(t) \frac{\alpha \sigma^{2}}{2} \int_{t}^{\infty} q^{2}(u) d u(1+\varepsilon(t))
$$

where $\varepsilon(t) \rightarrow \infty$ as $t \rightarrow \infty$.

Proof. Indeed, by (22), Lemma 16, and the equivalence $h(x) \sim \sigma^{2} x^{2} / 2$ as $x \rightarrow+0$, we have

$$
\begin{aligned}
q(t) & =P(t)-\alpha \int_{0}^{t} h(q(u)) P(t-u) d u=\alpha P(t) \int_{t}^{\infty} h(q(u)) d u-\alpha J(t) \\
& =\alpha P(t) \int_{t}^{\infty} h(q(u)) d u(1+o(1))=P(t) \frac{\alpha \sigma^{2}}{2} \int_{t}^{\infty} q^{2}(u) d u(1+\varepsilon(t)) .
\end{aligned}
$$

Proof of Theorem 2. Set

$$
y(t)=\int_{t}^{\infty} q^{2}(u) d u .
$$

Then (29) can be rewritten as follows:

$$
\sqrt{-y^{\prime}(t)}=P(t) \frac{\alpha \sigma^{2}}{2} y(t)(1+\varepsilon(t))
$$


or

$$
\begin{aligned}
-y^{\prime}(t) & =\left(P(t) \frac{\alpha \sigma^{2}}{2} y(t)\right)^{2}\left(1+\varepsilon_{1}(t)\right) \\
& =\frac{1}{t+1}\left(\frac{\gamma_{1}}{1-\alpha}\right)^{2}\left(\frac{\alpha \sigma^{2}}{2}\right)^{2} y^{2}(t)\left(1+\varepsilon_{2}(t)\right)=\frac{K_{1}}{t+1} y^{2}(t)\left(1+\varepsilon_{2}(t)\right)
\end{aligned}
$$

in view of (16) where

$$
K_{1} \stackrel{\text { def }}{=}\left(\frac{\gamma_{1}}{1-\alpha}\right)^{2}\left(\frac{\alpha \sigma^{2}}{2}\right)^{2}
$$

and $\varepsilon_{i}(t), i=1,2, \ldots$, denote functions vanishing as $t \rightarrow \infty$. Solving (30) under the initial condition

$$
y(0)=C_{0}^{-1} \stackrel{\text { def }}{=} \int_{0}^{\infty} q^{2}(u) d u
$$

we obtain

$$
y(t)=\left(C_{0}+K_{1} \int_{0}^{t} \frac{\left(1+\varepsilon_{2}(u)\right)}{u+1} d u\right)^{-1} \sim \frac{1}{K_{1} \ln t}, \quad t \rightarrow \infty .
$$

Substituting this equivalence into (29) we see that

$$
\begin{aligned}
q(t) & \sim P(t) \frac{\alpha \sigma^{2}}{2} \frac{1}{K_{1} \ln t}=\frac{2}{\alpha \sigma^{2}}\left(\frac{1-\alpha}{\gamma_{1}}\right)^{2} \frac{P(t)}{\ln t} \\
& \sim \frac{2}{\alpha \sigma^{2}} \frac{1-\alpha}{\gamma_{1}} \frac{1}{\sqrt{t} \ln t}=\frac{2(1-\alpha)}{\alpha \sigma^{2}} b \sqrt{2 \pi} \frac{1}{\sqrt{t} \ln t}=K^{2} \pi \frac{1}{\sqrt{t} \ln t}
\end{aligned}
$$

as $t \rightarrow \infty$. This relation proves Theorem 2 .

\section{BIBLIOGRAPHY}

1. S. Albeverio and L. V. Bogachev, Branching random walk in a catalytic medium. I. Basic equations, Positivity 4 (2000), no. 1, 41-100. MR.1740207 (2001a:60098)

2. S. Albeverio, L. V. Bogachev, and E. B. Yarovaya, Asymptotics of branching symmetric random walk on the lattice with a single source, C. R. Acad. Sci. Paris Ser. I Math. 326 (1998), no. 8, 975-980. MR 1649878 (99j:60126a)

3. K. B. Athreya and P. E. Ney, Branching Processes, Springer-Verlag, New York-Heidelberg, 1972. MR0373040(51:9242)

4. L. V. Bogachev and E. B. Yarovaya, A limit theorem for a supercritical branching random walk on $\mathbf{Z}^{d}$ with a single source, Russian Math. Surveys 53 (1998), no. 5, 1086-1088. MR1691192 (2000f:60128)

5. , Moment analysis of a branching random walk on a lattice with a single source, Dokl. Akad. Nauk 363 (1998), no. 4, 439-442. (Russian) MR1702745 (2000h:60079)

6. D. A. Dawson and K. Fleischmann, Longtime behavior of a branching process controlled by branching catalysts, Stochastic Process. Appl. 71 (1997), 241-257. MR1484162 (99c:60187)

7. W. Feller, An Introduction to Probability Theory and Its Applications, vol. II, John Wiley \& Sons, New York-London-Sydney, 1971. MR0270403 (42:5292)

8. K. Fleischmann, Superprocesses in catalytic media, Measure-Valued Processes, Stochastic Partial Differential Equations, and Interacting Systems (Montreal, PQ, 1992), CRM Proc. Lecture Notes, vol. 5, Amer. Math. Soc., Providence, RI, 1994, pp. 99-110. MR1278286 (95b:60103)

9. K. Fleischmann and J. F. Le Gall, A new approach to the single point catalytic super-Brownian motion, Probab. Theory Related Fields 102 (1995), no. 1, 63-82. MR1351711 (96g:60107)

10. F. M. J. Olver, Introduction to Asymptotics and Special Functions, Academic Press, NewYork-London, 1974. MR0435697 (55:8655) 
11. B. A. Sewastyanow, Verzweigungsprozesse, Akademie-Verlag, Berlin, 1974. MR0408018 $(53: 11785)$

12. V. A. Vatutin, Discrete limit distributions of the number of particles in a Bellman-Harris branching process with several types of particles, Theory Probab. Appl. 24 (1979), 509-520. MR0541363 (80j:60112)

Steklov Mathematical Institute, Gubkina Street 8, 117966, GSP-1 Moscow, Russia

E-mail address: vatutin@mi.ras.ru

Omsk Branch of Sobolev Institute of Mathematics, Pevtsova Street 13, 644099 Omsk, RUSSIA

E-mail address: topchij@iitam.omsk.net.ru

Faculty of Mathematics and Mechanics, Moscow State University, 119992, GSP-2 Moscow, RUSSIA

E-mail address: yarov@cardpl.msk.ru

Received 24/FEB/2003

Translated by THE AUTHORS 